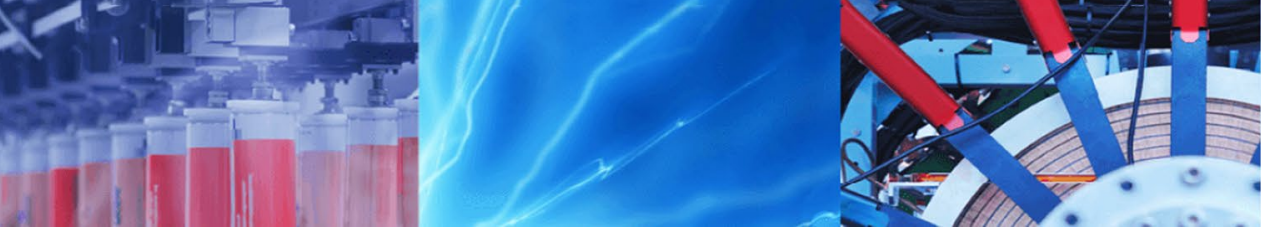

Research Article

\title{
Modeling of the scattering mechanisms of a two-dimensional Fermi electron gas in the heterostructure InAs/AISb/InAs
}

\author{
E. R. Burmistrov ${ }^{1}$ D $\cdot$ M. M. Afanasova ${ }^{1}$
}

Received: 18 November 2019 / Accepted: 28 July 2020 / Published online: 19 August 2020

(c) Springer Nature Switzerland AG 2020

\begin{abstract}
The article investigates the mechanism by ionized atoms carrier scattering at impurities heterostructure InAs/AISb with two occupied subbands. The aim of the article is to develop and complement the existing theory which fully describes the quantum mechanical processes in the structure of the study. Conducted transport time calculation showed that the proposed theory accurately describes the two-dimensional carrier scattering mechanism is donor impurity ions in the active layer of InAs. It was found that the lifetime of limiting the mobility of two-dimensional carrier in scattering by impurity ions is $10^{-12} \mathrm{~s}$.
\end{abstract}

Keywords Quantum well · The scattering on impurity atoms · Doped heterostructure · Transport time - The deployment of donor impurity ions · Elastic scattering

\section{Introduction}

In early works [1-3] was subjected to the relaxation time for such dominant scattering mechanisms carriers twodimensional electron gas (DEG) in the heterostructure InAs/AISb as scattering irregularities on the heteroboundary resulting in electron-electron scattering. It focused on the quantum intersubband relaxation time. In this regard, it has been suggested that the broadening of the Landau levels is the reason for limiting the Shubnikov-de Haas effect and small-angle scattering of two-dimensional media is defined.

The basic formulation of the problem of scattering of charge carriers in the impurity ions in the quantum limit and Howard Stern realized in [4]. In operation, the authors found that the impurity atoms form energy levels of the quantum size near the boundaries and permitted areas within the forbidden zone. Therefore, the impurity atoms move easily in the ionized state at low temperatures, which are a major source of disturbance in the heterostructure InAs/AISb.

The study of quantum mechanical calculation of the scattering potential and the density matrix has allowed to develop a theoretical model, and it gave high-quality results.

In this paper, heterostructure InAs/AISb is filled with the two subbands, which makes it possible to evaluate the relaxation time for a given scattering mechanism on each of the subbands [5]. The electrons in the doped samples InAs QW were supplied from two-layer tellurium (Te) located at a distance of $15 \mathrm{~nm}$ from the heterojunction InAs/AISb [2]. The band diagram of the heterostructure studied [3] is presented in Fig. 1.

One result was the creation of the theory with which it was possible to describe the behavior of two-dimensional media and give a numerical estimate of the quantum mechanical phenomena in the heterostructure InAs/AISb 1 and 2 filled with subzones. In this paper, we propose a new theoretical method for calculating the

E. R. Burmistrov, eugeni.conovaloff@yandex.ru; M. M. Afanasova, marinaaf-80@mail.ru| ${ }^{1}$ Ryazan State University Named for S. Esenin, 46 Svobody, Ryazan, Russia 390000. 


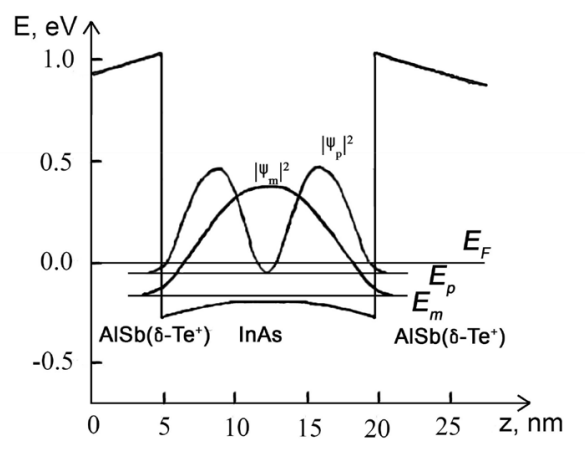

Fig. 1 Band diagram of the structure AISb $(\delta$-Te)/InAs/AISb $(\delta$-Te)

relaxation time. During the research, it was found that the method allows to make changes in its structure associated with the formulation of the problem conditions. In view of this particular method of calculation of the relaxation time, it is versatile and can be used to describe transfer processes in quantum wells with a more complicated form of the potential relief. It is suggested that the developed theory can be used for the investigation of two-dimensional transfer of carriers in quantum wells with an asymmetric probability distribution of the wave functions.

\section{Theoretical model}

Studied were grown by molecular beam epitaxy. The band diagram of the heterostructure AISb $(\delta-\mathrm{Te}) / \mathrm{InAs} /$ $\operatorname{AlSb}(\delta$-Te $)$ is shown in Fig. 1.

The isotropy of the two-dimensional Fermi surface makes it possible to use the apparatus of tensor calculus for the implementation of the mathematical model. For doped heterostructure InAs/AISb with two occupied subbands, relaxation time is determined by simplified kinetic equation of Boltzmann [6]

$$
\sum_{j} K_{i j} \tau_{j}=E_{F i}
$$

where $K_{i j}$ is the scattering component tensor characterizing the contribution of the first and second subbands in scattering processes with transport time $\tau_{t} ; E_{i}$ is the position of the energy subzones measured relative to the Fermi level $E_{F_{i}}$ and moreover

$E_{F_{i}}=E_{F}-E_{i}$.

In general, scattering of the tensor components is expressed through the formula [7]

$$
K_{i j}=\left(k_{F_{i}} / 2 \pi \hbar\right) \times\left[\int_{0}^{\pi}\left(k_{F_{i}} \delta_{i j} \sum\left|V_{i l}\left(q_{i l}\right)\right|^{2}-k_{F j}\left|V_{i j}\left(q_{i j}\right)\right|^{2} \cos \vartheta\right) d \vartheta\right]
$$

or the notation

$$
\begin{aligned}
K_{i j}^{1} & =k_{F_{i}}^{2} / 2 \pi \hbar \times \int_{0}^{\pi}\left|V_{i j}\left(q_{i j}\right)\right|^{2} d \vartheta, K_{i j}^{2} \\
& =k_{F_{i}} k_{F_{j}} / 2 \pi \hbar \times \int_{0}^{\pi} \cos \vartheta\left|V_{i j}\left(q_{i j}\right)\right|^{2} d \vartheta,
\end{aligned}
$$

where $k_{F_{i}}$ is the Fermi wave vector in $i_{\text {th }}$ subband; $k_{F_{j}}$ is the Fermi wave vector in $j_{\text {th }}$ subband; $\vartheta$ is the scattering angle; $\left|v_{i j}\left(q_{i j}\right)\right|^{2}$ is the squared matrix potential of scattering of a two-dimensional Fermi gas by impurity ions; $\hbar=1.055 \cdot 10^{-34} \mathrm{~J} \cdot \mathrm{s}$ is the Dirac constant; and $\delta_{i j}=\left\{\begin{array}{ll}1, & i=j \\ 0, & i \neq j\end{array}\right.$ is the delta Dirac function. The relation between the scattering tensors is given by the following expression

$K_{i j}=\delta_{i j} \sum_{i} K_{i l}^{1}-K_{i j}^{2}$.

Since the process of scattering on impurity atoms is elastic, the terms in the formula $K_{i j}^{2}$ are integrated to zero. We obtain the final formula for calculating the components of the tensor [7]

$K_{i j}=\delta_{i j} \sum_{l} K_{i l}^{1}=\left(k_{F_{i}}^{2} \delta_{i j} / 2 \pi \hbar\right) \times \int_{0}^{\pi} \sum_{l}\left|V_{i l}\left(q_{i l}\right)\right|^{2} d \vartheta$.

Given the entered symbols, relaxation time in scattering of impurity ions donor $\mathrm{N}_{T e}^{+}$in each of the subbands is determined by the following formulas [7]

$\tau_{0}^{-1}=\left(K_{00} K_{11}-K_{01} K_{10}\right) /\left(E_{F 0} K_{11}-E_{F 1} K_{01}\right)$,

$\tau_{1}^{-1}=\left(K_{00} K_{11}-K_{01} K_{10}\right) /\left(E_{F 1} K_{00}-E_{F 0} K_{10}\right)$,

where $q_{i j}=\left(\vec{k}_{F_{i}}^{2}+\vec{k}_{F_{j}}^{2}-2 \vec{k}_{F_{i}} \vec{k}_{F_{j}} \cos \vartheta\right)^{1 / 2}$ is the change in wave vector due to the elastic scattering from $\mathrm{i}^{\text {th }}$ to $\mathrm{j}^{\text {th }}$ subband. According to the rule Mattis total lifetime, limiting the mobility of two-dimensional carrier at a given scattering mechanism is given by

$\tau_{M}=\left(\tau_{0} N_{0}+\tau_{1} N_{1}\right) /\left(N_{0}+N_{1}\right)$,

where $N_{0}, N_{1}$ is the impurity concentration at the first and second subbands, respectively. Based on numerous articles in which the authors study the impurity distribution profile in the heterostructure $\operatorname{InAs} / \mathrm{AISb} / \operatorname{InAs}$, we fitted the 
donor and acceptor atom distributions by Gaussian law. In our work, we can use the corresponding approximation which consists in the fact that the impurity distribution profile is approximately subordinate to the Gauss law.

This is explained by the fact that a $\delta$-layer was formed as a result of doping of a donor impurity into the region of the InAs/AISb/InAs heterostructure barrier. In this regard, an impurity concentration gradient arises in depth with a characteristic maximum in the near-surface layer. Only the upper wings of impurity depth profiles were used for fitting because the lower wings of impurity depth profiles were distorted due to ion beam mixing processes known as knock-on effect. The experimental plot of the impurity concentration distribution profile taken from [3] clearly indicates the fact that we are talking about a Gaussian distribution (Fig. 2). In connection with the indicated features and experimental graphs, we can apply the corresponding Gaussian law

$N_{i}=N_{T e} / \sqrt{2 \pi \sigma_{i}^{2}} \times \exp \left(-(z-v)^{2} / 2 \sigma_{i}^{2}\right)$,

where $\sigma_{i}$ is the standard deviation coordinates and $v$ is the position doping layer; $N_{T e}$ is the impurity concentration in $\delta$-layer. Tensor components through calculate ratios

$$
\begin{aligned}
\left|V_{i j}^{i m p}\left(q_{i j}\right)\right|^{2}= & e^{4} / 2 \varepsilon_{0}^{2} \varepsilon_{s}^{2} q_{i j}^{2} \times \int d\left(z_{i}\right) \cdot N\left(z_{i}\right) \\
& \times\left(\sum_{n, m}\left(\varepsilon_{i j, n m}^{-1}\right) \cdot F_{n m}(q, z)\right)^{2} .
\end{aligned}
$$

Dielectric matrix describing the screening effect for various transitions between filled subbands $\left(\varepsilon_{i j, n m}\right)$ with potentials scattering is expressed by the formula

$V_{i j}\left(q_{i j}\right)=\sum_{n, m}\left(\varepsilon_{i j, n m}\right)^{-1} \times \tilde{V}_{n m}\left(q_{i j}\right)$,

where $V_{i j}\left(q_{i j}\right), \tilde{V}_{n m}\left(q_{i j}\right)$ are matrix element-screened and unscreened Coulomb potentials, respectively; $\varepsilon_{s}$ is the static dielectric constant; $\varepsilon_{0}$ is the dielectric constant; and $z_{i}$ is the position of the first and second subzones along the $z$-axis. In our case, dielectric matrix indexes change in range $i, j, n, m=0,1$. The form factor is given by

$$
\begin{aligned}
H_{i j, n m}\left(q_{i j}\right)= & \int_{0}^{\infty} \mathrm{d} z \int_{0}^{\infty} \mathrm{d} z^{\prime} \phi_{i}(z) \cdot \phi_{j}(z) \\
& \times \exp \left(-q_{i j}\left|z-z^{\prime}\right|\right) \cdot \phi_{n}\left(z^{\prime}\right) \cdot \phi_{m}\left(z^{\prime}\right) .
\end{aligned}
$$

The matrix element of the scattering potential by impurity ions is given by the following expression [8]

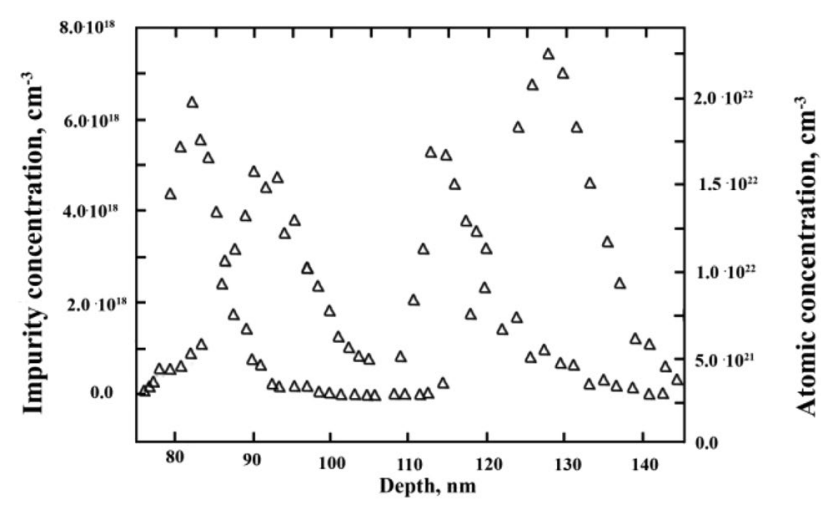

Fig. 2 Atomic distribution of heterostructure AISb/InAs/AISb from paper [3]. The distribution profile of the impurity of tellurium atoms is shown
Potential pattern distortion due to crystal lattice defects also greatly influences the nature of the collision of twodimensional electrons. Alloying heterostructure InAs/ AISb $\delta$-layer gives rise to an induced charge at the positive impurity ions Te. Rapidly changing potentials lead to the fact that it is necessary to take into account the effect of screening. To solve the problem of taking into account the shielding effect, we use the first-order perturbation theory in the framework of the screening Lindhardt and expand on the potential of wave function

$\psi_{n}=\frac{1}{\sqrt{V}} \exp (i \vec{k} \vec{r})+\frac{2 m e}{\hbar^{2} V \sqrt{V}} \sum \frac{\exp \left(i \overrightarrow{k^{\prime}} \vec{r}\right)}{\vec{k}^{2}-\bar{k}^{\prime 2}} \phi_{\vec{k}^{\prime}-\vec{k}^{\prime}}$

where $V$ is the volume studied structure generally and $\phi$ is the potential. By definition, the Fourier component $\phi_{k^{\prime}-k}$ is determined via the integral of the form

$\phi_{k^{\prime}-k}=\int \phi(\vec{r}) \times \exp \left(i\left(\vec{k}-\overrightarrow{k^{\prime}}\right) \vec{r}\right) d \vec{r}$. 
In linear approximation, induced charge is given by [9]

$$
\begin{aligned}
\rho^{\text {ind }}(\vec{r})= & 4 m \cdot\left(\frac{e}{\hbar V}\right)^{2} \sum_{k} f_{k} \sum_{\vec{k}^{\prime}}\left(\exp \left(i \cdot\left(\overrightarrow{k^{\prime}}-\vec{k}\right) \cdot \vec{r}\right) \cdot \varphi_{\overrightarrow{k^{\prime}}-\vec{k}}\right. \\
& +\frac{\exp \left(i \cdot\left(\overrightarrow{k^{\prime}}-\vec{k}\right) \cdot \vec{r}\right)}{\left(\vec{k}^{2}-\vec{k}^{\prime}\right)} \varphi_{\vec{k}^{\prime}-\vec{k}^{\prime}}^{\prime}
\end{aligned}
$$

where $f_{k}$ is the Fermi-Dirac equilibrium function. We introduce the replacement: $\bar{q}=\bar{k}^{\prime}-\bar{k}, \bar{Q}=\left(\overline{k^{\prime}}+\bar{k}\right) / 2-$ which are the difference and the sum of the pulses, respectively. By virtue of the reality of the Fourier component, the following condition is fulfilled for them $\varphi_{-\bar{q}}^{*}=\varphi_{\bar{q}}$. The expression for the induced charge takes the following form

$\rho^{\text {ind }}(\bar{r})=\frac{-2 m e^{2}}{\hbar^{2} V^{2}} \sum_{\bar{Q}} \sum_{\bar{q}}\left(f_{(\bar{Q}-\bar{q}) / 2}-f_{(\bar{Q}+\bar{q}) / 2}\right) \frac{\exp (i \bar{q} \bar{r})}{\bar{q} \bar{Q}} \varphi_{\bar{q}^{\prime}}$

where $\chi(\bar{q})=\rho^{\text {ind }}(\bar{q}) / \varphi_{\bar{q}}$ - is the static polarization. In our case (in the limit of slowly varying potentials $q<k_{F}$ and low temperatures $T \rightarrow 0$ ), the dielectric permeability is related to static polarization according to $[6]: \varepsilon(\bar{q})=1-4 \pi \chi(\bar{q}) / q^{2}$.

Therefore,

$$
\begin{aligned}
\varepsilon(\bar{q}) & =1-4 \pi \chi(\bar{q}) / q^{2}=1+\frac{\mathrm{e}^{2}}{\pi^{2} q^{2}} \int \frac{f_{(\bar{Q}-\bar{q}) / 2}-f_{(\bar{Q}+\bar{q}) / 2}}{\hbar^{2}(\bar{Q} \cdot \bar{q}) / m+\hbar \omega} \\
& =1+\frac{4 \pi e^{2}}{q^{2}} \int \frac{d \bar{Q}}{4 \pi^{3}} \frac{f_{\bar{Q}+\bar{q}}-f_{\bar{Q}}}{\varepsilon_{\bar{Q}+\bar{q}}^{0}-\varepsilon_{\bar{Q}}^{0}}=1+\frac{q_{s}}{q} H(q) \chi(q)
\end{aligned}
$$

We rewrite formula (18) in tensor notation

$\varepsilon_{\alpha, \beta}(q)=\delta_{\alpha} \delta_{\beta}+\frac{q_{s}}{q_{\alpha}} H_{\alpha, \beta}\left(q_{\alpha}\right) \chi_{\alpha}\left(q_{\alpha}\right)$,

where $\quad \alpha=i j, \quad \beta=n m ; \quad q_{s}=m^{*} e^{2} / 2 \pi \varepsilon_{s} \varepsilon_{0} \hbar^{2}, \delta_{\alpha} \delta_{\beta}$ $=\left\{\begin{array}{l}1, i=j, n=m \\ 0, i \neq j, n=m ; i=j, n \neq m ; i \neq j, n \neq m\end{array}\right.$-are the Kronecker symbols. Formula (19) is applicable in our case since the $\ln \mathrm{As} / \mathrm{AlSb} / \mathrm{InAs}$ heterostructure is considered in the limit of slowly varying potentials (adiabatic approximation) and in the limit of low temperatures.

Matrix static polarization and form factor are expressed, respectively [10]

$\chi_{i j}(q)=\left\{\begin{array}{l}1-\Theta\left(q_{i j}-2 k_{F_{i}}\right) \sqrt{\left(1-\left(2 k_{F_{i}} / q_{i j}\right)^{2}\right)}, i=j \\ \frac{q_{i j}^{2}+k_{0}^{2}-\sqrt{\left(q_{i j}^{2}+k_{0}^{2}\right)^{2}-\left(2 q_{i j} k_{F_{i}}\right)^{2}}}{2 q_{i j}^{2}}, k_{F_{i}} \leq k_{0}, \quad i \neq j \\ 1-\frac{\Theta\left(q_{i j}-k_{+}\right)}{q^{2}} \sqrt{\left(q_{i j}^{2}-k_{+}^{2}\right)\left(q_{i j}^{2}-k_{-}^{2}\right)}, k_{F_{i}}>k_{0}\end{array}\right.$ where $\varphi_{i}(z), \varphi_{j}(z)$ are subzones of the wave functions; $\Theta(x)$ is the Heaviside step function; and $m_{e}^{*}$ is the effective mass of the electron. The effect of changing the mass of charge carriers depending on the position inside the quantum well (PDM) is not investigated in this article although this effect is very important in the heterostructure. We do not consider it since a quantum well based on the $\mathrm{AlSb} / \mathrm{InAs} / \mathrm{AlSb}$ compound has a rectangular potential relief. This fact follows from a self-consistent solution of the Schrödinger equation and Poisson electroneutrality and is also confirmed in [10]. It is worth noting that the bending of the zones in the barrier and in the quantum well is insignificant. The potential changes stepwise at the heterointerface, while inside the pit it remains constant. Recall that AISb/InAs/AISb heterostructures were considered under conditions of quantizing magnetic fields. This feature sharply restricts the motion of a two-dimensional electron gas. The active InAs layer consisted of two filled size quantization subbands. The squared modulus of the wave function had a maximum in the middle of the energy level. This can be seen from Fig. 1. Therefore, the electron density is concentrated in the center of the quantum well. All these features lead to the fact that charge carriers are localized in the center of the quantum well and are sharply limited in motion [11]. This makes it impossible to consider ballistic transport in a heterostructure and leads to the conclusion that the effective mass changes stepwise only at the heterointerface. Due to the resonant interaction, quantum time characterizes the entire system of twodimensional electrons in the InAs layer and is a measure of collective movement [12]. A two-dimensional electron gas is located in the center of the quantum well, and the potential relief has a rectangular symmetrical shape and changes stepwise at the heterointerface. The square of the wave function has a maximum in the center of the potential well. Therefore, it makes no sense to consider the PDM effect in this heterostructure.

The value of the effective electron mass in this paper is constant and equal to $m_{e}^{*}=0.033 \cdot m_{e}=0.033 \times 1.6 \cdot 10^{-19} \mathrm{C}$. $k_{0}^{2}=2 m^{*}\left(E_{1}-E_{0}\right) / \hbar^{2} ; k_{+}=\left|k_{F_{0}}+k_{F_{1}}\right| ; k_{-}=\left|k_{F_{0}}-k_{F_{1}}\right|$.

In the quantum well, the symmetry of the wave functions leads to the fact that the form factor (13) is different from zero only in the case where the sum of the indices $i, j, n$ and $m$ is even. We write the matrix elements determining the scattering in the first subband. Matrix dielectric permeability is of the form

$$
\varepsilon_{00,00}\left(q_{00}\right)=1+\frac{m^{*} e^{2}}{2\left(\sqrt{\left.2 k_{0}^{2}-2 k_{0}^{2} \cos \vartheta\right)} \pi \varepsilon_{s} \varepsilon_{0} \hbar^{2}\right.} H_{00,00}\left(q_{00}\right) \cdot \chi_{00}\left(q_{00}\right) .
$$


In this case when $i=j$, the matrix static polarization is expressed by the formula

According to the mathematical definition of the Heaviside function

$\Theta(x)=\left\{\begin{array}{l}0, x<0 \\ 1, x>0\end{array}\right.$,

in terms of this task,

$\Theta\left(q_{00}-2 k_{F 0}\right)=\left\{\begin{array}{l}0, q_{00}-2 k_{F 0}<0 \\ 1, q_{00}-2 k_{F 0}>0\end{array}\right.$ ，

where $q_{00}=\sqrt{2 k_{F_{0}}^{2}(1-\cos \vartheta)}=2 k_{F_{0}} \sin (\vartheta / 2)$ and

$\Theta\left(q_{00}-2 k_{F_{0}}\right)=\left\{\begin{array}{l}0,2 k_{F_{0}} \sin (\vartheta / 2)<2 k_{F_{0}} \\ 1,2 k_{F_{0}} \sin (\vartheta / 2)>2 k_{F_{0}}\end{array}\right.$.

Consequently, a step function $\Theta=0$ and $\chi_{00}\left(q_{00}\right)=1$. The redistribution of small values $q$ form factor $H_{00,00}\left(q_{00}\right)=1$. Given the findings, obtained formulas and formulas take the form

$\varepsilon_{00,00}\left(q_{00}\right)=1+\frac{m^{*} e^{2}}{4 k_{F_{0}} \sin (\vartheta / 2) \pi \varepsilon_{s} \varepsilon_{0} \hbar^{2}}$

$\left|V_{o o}^{i m p}\left(q_{00}\right)\right|^{2}=\frac{e^{4}}{2 \varepsilon_{0}^{2} \varepsilon_{s}^{2} 2 k_{F_{0}} \sin (\vartheta / 2)} \times \int N\left(z_{1}\right) d z_{1} \cdot\left(\left(\varepsilon_{00,00}\right)^{-1}\right)^{2}$.

Considering the scattering process in the second subband, we can write the matrix similar to the dielectric constant and the potential scattering matrix in the form of
$\chi_{00}(q)=1-\Theta\left(q_{00}-2 k_{F_{0}}\right) \sqrt{1-\left(2 k_{F_{0}} / q_{00}\right)^{2}}$.

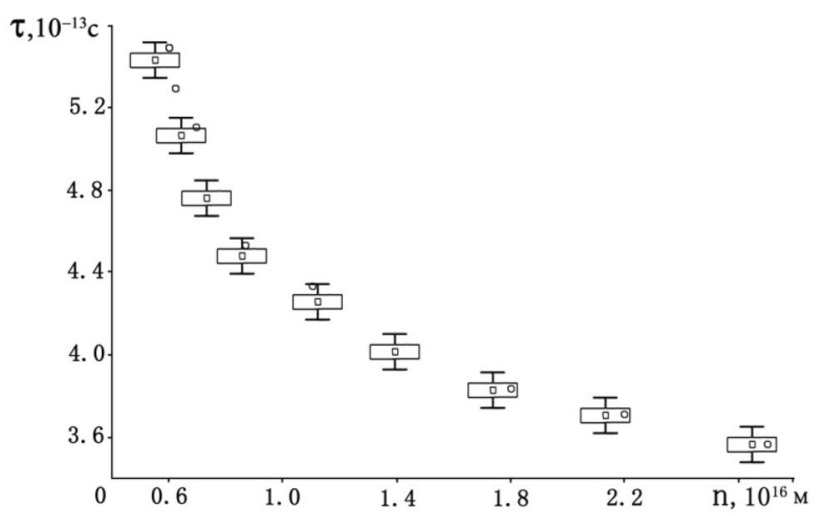

Fig. 3 Transport time dependence of the concentration of impurities in Te heterostructure $\mathrm{AlSb}(\delta$-Te)/InAs/AISb $(\delta$-Te)

$$
\begin{aligned}
q_{10}= & \sqrt{\left(k_{F_{1}}^{2}+k_{F_{0}}^{2}-2 k_{F_{1}} k_{F_{0}} \cos \vartheta\right)} \\
= & \sqrt{k_{F_{1}}^{2}\left(1+\eta^{2}-2 \eta \cos \vartheta\right)} \approx k_{F_{1}} \sqrt{(1-2 \eta \cos \vartheta) \approx} \\
& \approx k_{F_{1}}-k_{F_{0}} \cos \vartheta .
\end{aligned}
$$

Substituting all the previously obtained expressions into formula (7), we obtain the relaxation time in the first subzone

$$
\begin{aligned}
1 / \tau_{0}= & \frac{\frac{k_{F_{0}}^{2}}{2 \pi \hbar} \int_{0}^{\pi}\left|V_{00}\left(q_{00}\right)\right|^{2} \mathrm{~d} \theta \times \frac{k_{F_{1}}^{2}}{2 \pi \hbar} \int_{0}^{\pi}\left|V_{11}\left(q_{11}\right)\right|^{2} \mathrm{~d} \theta}{\left(E_{F}-E_{0}\right) \frac{k_{F_{1}}^{2}}{2 \pi \hbar} \int_{0}^{\pi}\left|V_{11}\left(q_{11}\right)\right|^{2} \mathrm{~d} \theta-\left(E_{F}-E_{1}\right) \frac{k_{F_{0}}^{2}}{2 \pi \hbar} \int_{0}^{\pi}\left|V_{01}\left(q_{01}\right)\right|^{2} \mathrm{~d} \theta} \\
& -\frac{\frac{k_{F_{0}} k_{F_{1}}}{2 \pi \hbar} \int_{0}^{\pi}\left|V_{01}\left(q_{01}\right)\right|^{2} \mathrm{~d} \theta \times \frac{k_{F_{F}} k_{F_{1}}}{2 \pi \hbar} \int_{0}^{\pi}\left|V_{10}\left(q_{10}\right)\right|^{2} \mathrm{~d} \theta}{\left(E_{F}-E_{0}\right) \frac{k_{F_{1}}^{2}}{2 \pi \hbar} \int_{0}^{\pi}\left|V_{11}\left(q_{11}\right)\right|^{2} \mathrm{~d} \theta-\left(E_{F}-E_{1}\right) \frac{k_{F_{0}}^{2}}{2 \pi \hbar} \int_{0}^{\pi}\left|V_{01}\left(q_{01}\right)\right|^{2} \mathrm{~d} \theta}
\end{aligned}
$$

$\varepsilon_{11,11}\left(q_{11}\right)=1+\frac{m^{*} e^{2}}{4 k_{F_{1}} \sin (\vartheta / 2) \pi \varepsilon_{s} \varepsilon_{0} \hbar^{2}},\left|V_{11}^{i m p}\left(q_{11}\right)\right|^{2}=\frac{e^{4}}{2 \varepsilon_{0}^{2} \varepsilon_{s}^{2} 2 k_{F_{1}} \sin (\vartheta / 2)} \int N\left(z_{1}\right) d z_{1}\left(\left(\varepsilon_{11,11}\right)^{-1}\right)^{2}$,

$\varepsilon_{10,10}\left(q_{10}\right)=\frac{m^{*} e^{2}}{2 q_{10} \pi \varepsilon_{s} \varepsilon_{0} \hbar^{2}} H_{10,10}\left(q_{10}\right),\left|V_{10}^{i m p}\left(q_{10}\right)\right|^{2}=\frac{e^{4}}{2 \varepsilon_{0}^{2} \varepsilon_{s}^{2}\left(q_{10}\right)^{2}} \int N\left(z_{1}\right) d z_{1}\left(\left(\varepsilon_{10,10}\right)^{-1}\right)^{2}$,

$\varepsilon_{01,01}\left(q_{01}\right)=\frac{m^{*} e^{2}}{2 q_{01} \pi \varepsilon_{s} \varepsilon_{0} \hbar^{2}} H_{01,01}\left(q_{01}\right),\left|V_{01}^{i m p}\left(q_{01}\right)\right|^{2}=\frac{e^{4}}{2 \varepsilon_{0}^{2} \varepsilon_{s}^{2}\left(q_{01}\right)^{2}} \int N\left(z_{0}\right) d z_{0}\left(\left(\varepsilon_{01,01}\right)^{-1}\right)^{2}$

A similar expression can be obtained for the second subzone. The total time $\left(\tau_{M}\right)$ should be calculated based on expression (8). 
Table 1 Analytical and theoretical data collected during the study, for a series of samples of heterostructures $\operatorname{AISb}(\delta$-Te $) / \operatorname{InAs} / \mathrm{AISb}(\delta$-Te $)$

\begin{tabular}{|c|c|c|c|c|c|c|c|}
\hline \multirow{2}{*}{$\begin{array}{l}\text { A series } \\
\text { of sam- } \\
\text { ples }\end{array}$} & \multicolumn{2}{|c|}{ Analytical calculation } & \multicolumn{5}{|c|}{ Theoretical calculation } \\
\hline & $\tau_{t}, 10^{-12} \mathrm{~s}$ & $N_{T e^{\prime}}, 10^{18} \mathrm{~cm}^{-3}$ & $\tau_{M^{\prime}}, 10^{-12} \mathrm{~s}$ & $N^{m}, 10^{18} \mathrm{~cm}^{-3}$ & $N^{p}, 10^{17} \mathrm{~cm}^{-3}$ & $\tau_{t}^{m}, 10^{-10} s$ & $\tau_{t}^{p}, 10^{-10} \mathrm{~s}$ \\
\hline 1 & 1.90 & 0.86 & 1.78 & 0.01 & 0.02 & 1.56 & 0.82 \\
\hline 2 & 0.71 & 1.50 & 0.57 & 0.22 & 0.18 & 1.12 & 0.74 \\
\hline 3 & 0.41 & 2.00 & 0.39 & 0.54 & 1.30 & 0.86 & 0.53 \\
\hline 4 & 0.29 & 2.40 & 0.20 & 0.87 & 2.29 & 0.47 & 0.28 \\
\hline
\end{tabular}

Table 2 Calculated lifetime of carriers in the heterostructure DEG $\operatorname{AlSb}(\delta$-Te)/InAs/AISb $(\delta$-Te)

\begin{tabular}{llll}
\hline $\begin{array}{l}\text { A series of } \\
\text { samples }\end{array}$ & $\tau_{t}^{m p}, 10^{-14} \mathrm{~s}$ & $N^{m}, 10^{18} \mathrm{~cm}^{-3}$ & $N^{p}, 10^{17} \mathrm{~cm}^{-3}$ \\
\hline 1 & 1.85 & 0.01 & 0.02 \\
2 & 0.64 & 0.22 & 0.18 \\
3 & 0.38 & 0.54 & 1.30 \\
4 & 0.26 & 0.87 & 2.29 \\
\hline
\end{tabular}

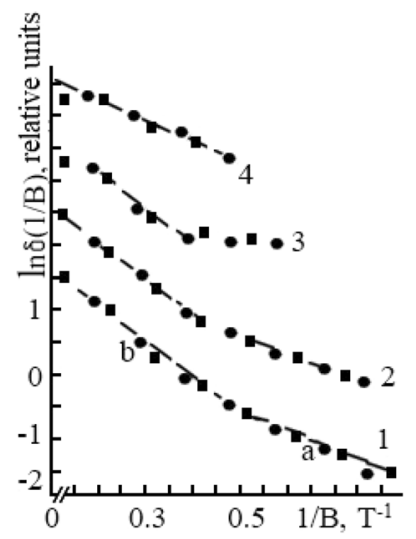

Fig. 4 Dependence Shubnikov-de Haas reverse magnetic field for the samples at the following temperatures $(T, K): 1-4.2,2-10.2$, 3-20.2, 4-28.6

\section{The discussion of the results}

The theoretical study of the magnetic field dependence graphs Shubnikov-de Haas (Fig. 4) reverse magnetic field able to analytically determine the relaxation time of two-dimensional electrons in the active layer of $\ln A s \tau_{t}$. The relaxation times $\tau_{t}$ were obtained in [5] by approximating the linear sections shown in Fig. 3 and determining their slopes. From theoretical calculations taking into account the relaxation of the two filled subbands was obtained for the ground $\tau_{t}^{m}$ and excited $\tau_{t}^{p}$ the total time of life $\tau_{M}$ (Table 1). The concentrations of impurities in the ground $N^{m}$ and excited $N^{p}$ subband obtained in the study theoretical methods of calculation are shown in Table 2.

In the course of the dependence of the transport time of the total impurity concentration considering two subbands (Fig. 3), the theoretical relaxation time values of the total concentration of positive donor subbands are plotted with the help of mathematical numerical data processing methods. Analytical and theoretical data were compared to assess the reliability of the values obtained by graphical methods. Figure 3 shows two graphs. Solid markers represent theoretical values $\tau_{M}(N)$; hollow markers represent analytical $\tau_{t}(N)$ that lie within the confidence interval of $15 \%$. Figure 3 shows that the penetration of the experimental points on the chart within the analytical values of the confidence interval indicates a high level of agreement between theory and experiment. Analyzing Fig. 3, we can conclude that the dependence of the relaxation time of the total impurity concentration in the heterostructure InAs/AISb obeys an exponential law. This fact is consistent with the fact that the dependence of the experimental values of the transport time of the concentration of positive donors as described by the exponential law. Qualitative analysis chart 1 leads to the conclusion that with the increase in the impurity concentration in the samples for transport lifetime, the mobility of two-dimensional carriers decreases. At the same time, with a decrease in concentration of the impurity contribution excited subband scattering mechanism of carriers in the two-dimensional electron gas by impurity ions Te decreases the total time of life increases exponentially [13]. Theoretical investigation established that for all samples, the condition $\tau_{p}<\tau_{m}$. This result is expected because the probability maxima of a square wave function of an electron in a quantum well are located in the second subarea and located closer to the heteroboundary than maxima of the electron wave function ground subband. Therefore, DEG excited carriers in the subband are in a stronger action field ion donor impurity and first come into elastic scattering mechanism.

The evaluation of the relaxation time in scattering of the two-dimensional electron gas by impurity ions with respect to this heterostructure InAs/AISb may be obtained from the simplification [14] 
$\tau_{t}^{i m p}=\frac{\left(2 k_{F_{m, p}} L\right)^{2} \times 64 \pi \hbar^{3} \varepsilon^{3}\left(2 \pi n_{s}\right)^{3 / 2}}{e^{4} m^{*} N_{T e} k_{F}^{2}\left(\frac{1}{L^{2}}-\left(L_{1}+W_{1}\right)^{-2}\right)}$,

$L$ is the width of the quantum well; $W_{1}$ is the width of the space charge; $k_{F_{m, p}}$ is the Fermi wave vector; $d_{s p}$ is the thickness of the spacer; and $z_{0}$ is the distance from the center of the electron wave function to the heteroboundary, wherein the impurity atoms are removed from a twodimensional electron gas at distance $\xi$

$L_{1}<\xi<R$,

where

$L_{1}=z_{0}+d_{s p}, R=L_{1}+W_{1}$.

Results of calculations $\tau_{t}^{i m p}$ performed based on formula (34) are shown in Table 2.

Comparing the data in Tables 1 and 2 shows that the relaxation time $\tau_{t}^{i m p}$ calculated based on Formula (34) is at least close to this accuracy $\tau_{t}$ than the values calculated using a matrix method $\tau_{M}$. This inconsistency suggests that the given formula does not take into account the screened Coulomb potential [15-17]. This inconsistency suggests that the given formula does not account for the screened Coulomb potential. Totally considering the screening medium dimensional electron gas in this case is difficult. However, one can get a good enough estimate using the formula for the radius of action of the field of the impurity ion in place of the disposition $[6,17]$

$r^{*}=\frac{1}{\sqrt{\left(\frac{4 \pi e^{4}}{v}\right) \times \int\left(\frac{-\partial f_{0}}{\partial \varepsilon}\right) g(\varepsilon) d \varepsilon}}$,

where $f_{0}(\varepsilon)$ is the equilibrium electron distribution function; $g(\varepsilon)$ is the density of states; and $v$ is the dielectric constant. In the Born approximation when $|U|<<\frac{\hbar^{2}}{m a^{2}}$ from Eq. 37, we obtain the screening radius

$r^{*}=\sqrt{v k_{0} T / 4 \pi e^{2} n}$

where $a$ is the radius of the potential; $m$ is the incident particle mass; and $U$ is the incident particle mass. The potential at a point lagging by arbitrarily dislocated ion donor impurity at a distance $r$ is given by the formula

$\varphi(r)=\frac{e}{v r} \exp \left(-r / r^{*}\right)$,

where the scattering potential is given by

$U(r)=\frac{e^{2}}{v r} \exp \left(-r / r^{*}\right)$.

\section{Overview of the quantum intersubband relaxation time in the heterostructure InAs/AISb}

Accounting for two filled subbands leads to additional effects associated with carriers of two-dimensional electron gas in the heterostructure. One of these effects is the intersubband scattering two-dimensional media. In our case that is in conditions of strong magnetic fields, the total relaxation time quantum will be formed by two independent channels: intra- and intersubband relaxation. To confirm this assumption, let us present graphs of the dependences of the amplitude of the Shubnikov-de Haas oscillations on the inverse magnetic field $[9,10]$. The heterostructure $\mathrm{AISb}(\delta$-Te)/InAs/( $\delta$-Te)AISb dynamics broadening of quantization depends on the scattering frequency DEG roughness heterojunction InAs/AISb which is most intensively implemented at sites 1 and 2 (Fig. 4).

This gives the reason to believe that in the strong magnetic fields of the slope sections $(a, b)$ is proportional Landau time and is mainly characterized by intersubband transitions for which $\tau \sim 10^{-15} \mathrm{~s}$. In Sects. 3, 4, dynamics Landau quantization reduced and Landau time is equal to $\sim 10^{-14} \mathrm{~s}$; the scattering is determined by roughness DEG $[18,19]$. Thus, it can be argued that the total relaxation time is formed taking into account the intra- and interband scattering mechanisms $[1,3,6]$.

One of the results of scientific research became a calculation time of intersubband scattering of electrons in the InAs active layer. In the face of competing intersubband, relaxation time of the scattering is determined by the equation

$\tau^{m p} \cong \frac{\tau^{*} \tau^{\text {intra }}}{\left(\tau^{\text {intra }}-\tau^{*}\right)}$

where $\tau^{*}$ is the total relaxation time considering intrasubband $\left(\tau^{\text {intra }}\right)$ and intersubband $\left(\tau^{\text {inter }}\right)$ scattering. Intrasubband relaxation time is generated in view of two independent scattering mechanisms; therefore,

$\frac{1}{\tau^{\text {inter }}}=\frac{1}{\tau_{q}^{m}}+\frac{1}{\tau_{q}^{p}}$

where $\tau_{q}^{m}$ and $\tau_{q}^{p}$ are the quantum relaxation time at the fundamental and excited subbands; $\tau^{\text {inter }}$ is the quantum intersubband relaxation time; and $\tau^{\text {intra }}$ is the quantum relaxation time intrasubband. The calculation results are presented in Table 3.

In Table 3, the following notations are introduced: $n_{m}$ - is the concentration of two-dimensional carriers in the main subzone; and $n_{p}$-is the concentration of two-dimensional carriers in the excited subband. The values $\left(n_{m}, n_{p}\right)$ are considered given, and their numerical values for a series 
Table 3 Theoretical values of the intersubband relaxation time of the heterostructure $\ln \mathrm{As} / \mathrm{AlSb}$

\begin{tabular}{|c|c|c|c|c|c|}
\hline \multirow{2}{*}{$\begin{array}{l}\text { A series of } \\
\text { samples }\end{array}$} & \multicolumn{2}{|c|}{ Analytical calculation } & \multicolumn{3}{|c|}{ Theoretical calculation } \\
\hline & $n_{m} t, 10^{16} \mathrm{~m}^{-2}$ & $n_{p^{\prime}} 10^{16} \mathrm{~m}^{-2}$ & $\tau^{m p}, 10^{-15} \mathrm{~s}$ & $\tau_{q}^{p}, 10^{-12} \mathrm{~s}$ & $\tau_{q}^{m}, 10^{-12} \mathrm{~s}$ \\
\hline 1 & 1.8 & 0.6 & 5.543 & 0.566 & 1.894 \\
\hline 2 & 2.2 & 1.0 & 4.606 & 0.476 & 1.839 \\
\hline 3 & 2.7 & 1.1 & 3.989 & 0.400 & 1.198 \\
\hline 4 & 3.6 & 0.6 & 3.678 & 0.413 & 1.101 \\
\hline
\end{tabular}

which are ionized impurity atoms Te. Scientific research could complement and enhance the existing theory on the mechanism of carrier scattering DEG impurity atoms in the heterostructure AISb $(\delta$-Te)/InAs/AISb $(\delta$-Te). It was possible to estimate the time and intersubband scattering speed. Engaging electron intersubband scattering processes requires additional energy and hence an increase in the pulse. In this study, samples were exposed to strong magnetic fields. Consequently, the total relaxation time quantum is formed by two channels: intra- and intersubband scattering. In this case, LL broadening largely determines the intersubband transitions; hence, the time of such transitions is less than the time of intrasubband of the order of $10^{-15} \mathrm{~s}$. This explains the fact that the rate of electron-electron intersubband interactions exceeds the absolute value of the electron-electron intrasubband.

Theoretically determined lifetime dimensional charge carriers at a given scattering mechanism are of the order of $10^{-12}$ seconds. During the obtained numerical values of the impurity concentration in the subzones and qualitatively analyzed transport time data obtained from the theory and experiment. Conducted DEG transport time calculation taking into account the filling of two subbands in the InAs/AlSb provided an opportunity to study the kinetics of the two-dimensional electrons, and also to estimate the contribution of each subband to the total relaxation time. It was found that the developed theory with high reliability under study describes the effects of the two-dimensional carriers in heterostructure InAs/AISb and can serve as a basis for the study of transport processes in heterostructures with different types of potential relief.

Used in quantum mechanical calculation allowed under the impact of movement of electrons of different intersystem evaluate factors fluctuation changing parameters characterizing transport processes in the heterostructure $\operatorname{AlSb}(\delta$-Te)/InAs/AISb $(\delta$-Te).

From the data in Tables 1 and 2, it is seen that in the structures with several subbands filled with increased impurity concentration and growth of the principal quantum number of samples in the lifetime, the mobility of carriers decreases.

In the course of scientific research, only elastic scattering mechanisms are considered as the inelastic scattering

It is established that one of the dominant mechanisms of heterostructure $\mathrm{AlSb}(\delta$-Te)/InAs/AISb $(\delta$-Te) is the scattering of carriers in the two-dimensional electron gas

\section{SN Applied Sciences}


by polar optical phonons which are negligible at low temperatures. The method takes into account the shielded and unshielded Coulomb potential. This assessment is given involving screening Lindhardt theory. One of the main results of the test developed theory on the mechanism of scattering on impurity ions was $90 \%$ agreement between the experimental and theoretical data.

\section{Compliance with ethical standards}

Conflict of interest The authors declare that they have no conflict of interest.

\section{References}

1. Kadushkin VI, Tsahhaev FM (2000) Intersubband relaxation of 2D electrons in algaas(si)/gaas heavily doped heterojunction. Phys. Low Dim. Struct. 1:2

2. Afanasova MM (2006) Scattering mechanisms in heavily doped structures $\mathrm{AlSb}(\delta-\mathrm{Te}+) / \mathrm{InAs} / \mathrm{AISb}(\delta-\mathrm{Te}+)$ with a degenerate 2D electron gas. In: Electronics: collection of scientific works. Ryazan. RSREU. 108-120

3. Burmisrov ER (2020) Scattering of charge carriers by impurity ions in InAs/AISb heterostructure. Russian Phys J 63(3):1-11

4. Stern F, Howard WE (1967) Properties of semiconductor surface inversion layers in the electric quantum limit. Phys Rev 163(7):816-835

5. Burmistrov ER (2020) The study of the quantum relaxation time in the heterostructure INAS/ALSB with one and two filled subbands. Nanostructures. Math Phys Model 32(9):1-11

6. Landau LD, Lipchitz EM (1963) Quantum mechanics. Non - relativistic theory. Elesiver, Amsterdam, pp 362-363

7. Ando T, Fowler A, Stern F (1982) Electronic properties of twodimensional systems. Rev Mod Phys 54(2):437
8. Ando T (1978) High field magnetotransport IN GaAs/AIGaAs heterojunctions and SI mosfets. J Phys Soc Jpn 44:475-773

9. Afanasova MM, Stepanov VA (2012) Intermodulation of transverse magnetoresistance oscillations in structures with strong intersubband interaction. Nanostruct Math Phys Model 3(6)

10. Krishtopenko SS, Ikonnikov AV, Sadofyev YG et al (2015) Effect of electron-electron interaction on cyclotron resonance in highmobility InAs/AISb quantum wells. J Appl Phys 117(11):112813

11. Fletcher R, Zaremba E, D'Lorio M (1988) Investigation of subband-edge disorder in a two-dimensional electron gas using magnetic depopulation. Phys Rev B 38:7866

12. Price PJ (1981) Two-dimensional electron transport in semiconductor layers. Phonon scattering. Ann Phys 133:217-239

13. Jogai B (1995) Candela-class high-brightness InGaN/AIGaN doubleheterostructure blue-light-emitting diodes. Appl Phys Lett 66(4):436

14. Protasov D, Zhuravlev K (2017) The mobility of two-dimensional electron gas in DA-pHEMT heterostructures with various width. Sol St Electron 129:66

15. Kalna K, Mosko M (1996) Intersubband electron-electron scattering in asymmetric quantum wells designed for far-infrared emission. Phys Rev 54:17730

16. Kinsler $P$, Harrison $P(1998)$ Intersubband electron-electron scattering in asymmetric quantum wells designed for far-infrared emission. Phys Rev 58:4771

17. Moiseev K, Ivanov E, Zegrya G (2006) Electroluminescence in $\mathrm{p}-\mathrm{InAs} / \mathrm{AISb} / \mathrm{InAsSb} / \mathrm{AISb} / \mathrm{p}(\mathrm{n})$-GaSb type II heterostructures with deep quantum wells at the interface. Appl Phys Lett 88:132102

18. Vurgaftman I, Meyer J, Ram-Mohan L (2001) Band parameters for III-V compound semiconductors and their alloys. Appl Phys 89(11):5815

19. Smet J, Fonstad G, Hu Q (1996) Far-infrared intersubband luminescence from quantum cascade sources. Appl Phys 79(12):9305

Publisher's Note Springer Nature remains neutral with regard to jurisdictional claims in published maps and institutional affiliations. 